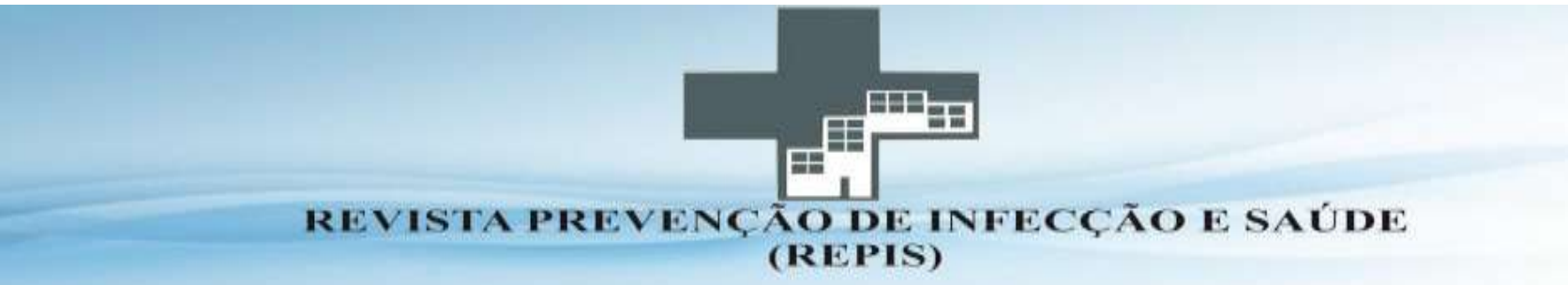

\title{
REFLEXIVO
}

\section{Formação do estudante de enfermagem sobre o controle de infecção na estratégia saúde da família: uma análise reflexiva}

Nursing student training on infection control in family health strategy: a reflexive analysis Formación del estudiante de enfermería sobre el control de infección en la estrategia de salud de la familia: un análisis reflexivo

Jardel Nascimento da Cruz ${ }^{1}$, Beatriz Carvalho Frota ${ }^{2}$, Francisco Marcio Nascimento da Cruz ${ }^{3}$, Francileia Nogueira Albino Calland ${ }^{4}$, Carlos Henrique Ribeiro Lima ${ }^{5}$, Camila Aparecida Pinheiro Landim Almeida ${ }^{6}$

\begin{abstract}
Objective: to carry out a reflection about the training of the nurse in the control of infection in the Strategy in the Family Health Strategy, in the Municipality of Teresina, Piauí. Method: it is a reflexive analysis, supported by theoretical references about the nursing student's training on infection control in the family health strategy Development: the knowledge acquired during graduation expresses the constant importance to perform a good functioning in the health institution, sought to consolidate the importance of nursing student training on the infection control in the family health strategy, providing the measures and conduct for professional action. Final considerations: The study may contribute to the context of continuing education in the nursing student's education to deal with infection control, enabling the construction of a discussion agenda, as well as new theoretical productions that imply the transformation of conceptions and practices generating new knowledge and learning.
\end{abstract}

Descriptors: Prevention e control; Nursing; Infection; Family Health Strategy.

\section{RESUMO}

Objetivo: realizar uma reflexão sobre a formação do Enfermeiro no controle de infecção na estratégia na Estratégia Saúde da Família, no Município de Teresina, Piauí. Métodos: trata-se de uma análise reflexiva, apoiada em referenciais teóricos sobre a formação do estudante de enfermagem sobre o controle de infecção na estratégia saúde da família. Desenvolvimento: os conhecimentos adquiridos durante a graduação exprimem as constantes importâncias para desempenhar um bom funcionamento na instituição de saúde, procurou-se consolidar a importância da formação dos estudantes de enfermagem sobre o controle de infecção na ESF, proporcionando as medidas e condutas para atuação profissional. Considerações finais: o estudo poderá contribuir no contexto da educação permanente na formação do estudante de enfermagem para lidar com o controle de infecção, possibilitando a construção de uma pauta de discussões, além de novas produções teóricas que implicam na transformação de concepções e práticas gerando novos conhecimentos e saberes.

Descritores: prevenção e controle; Enfermagem; Infecção; Estratégia Saúde da Família.

\section{RESUMÉN}

Objetivo: realizar una reflexión sobre la formación del enfermero en el control de infección en la estrategia en la Estrategia Salud de la Familia, en el Municipio de Teresina, Piauí. Método: se trata de un análisis reflexivo, apoyado en referenciales teóricos sobre la formación del estudiante de enfermería sobre el control de infección en la estrategia salud de la familia. Desarrollo: los conocimientos adquiridos durante la graduación expresan las constantes cantidades para desempeñar un buen funcionamiento en la institución de salud, se procuró consolidar la importancia de la formación de los estudiantes de enfermería sobre el control de infección en la ESF, proporcionando las medidas y conductas para actuación profesional. Conclusión: La toxoplasmosis es un serio problema de salud pública en Brasil y en el mundo, comprender sus manifestaciones clínicas en individuos inmunocompetentes se convierte en una herramienta útil para los equipos multiprofesionales a nivel hospitalario. Consideraciones finales: el estudio podrá contribuir en el contexto de la educación permanente en la formación del estudiante de enfermería para lidiar con el control de infección, posibilitando la construcción de una pauta de discusiones, además de nuevas producciones teóricas que implican en la transformación de concepciones y prácticas generando nuevos conocimientos y aprendizaje.

Descriptores: prevención e control; Enfermería; Infeccion; Estrategia de Salud Familiar.

1Discente de Enfermagem. Centro Universitário UNINOVAFAPI. E-mail: jardelnascimentoenfermagem14@gmail.com

2 Discente de Enfermagem. Centro Universitário UNINOVAFAPI. Teresina/PI, Brasil. E-mail: beatriz.frota@hotmail.com

${ }^{3}$ Discente de Enfermagem. Centro Universitário UNINOVAFAPI. Teresina/PI, Brasil. E-mail: calebre12345@gmail.com

${ }^{4}$ Educadora Física. Mestre. Docente. Centro Universitário UNINOVAFAPI. E-mail: fcalland@gmail.com.

5 Nutricionista. Mestrando em Saúde da Família. Centro Universitário UNINOVAFAPI. Teresina/PI, Brasil. E-mail: carlosnutri@hotmail.com.br

${ }^{6}$ Enfermeira. Doutora, Docente do Programa de Mestrado Profissional em Saúde da Família. Centro Universitário UNINOVAFAPI. Email: camila@uninovafapi.edu.br 
Formação do estudante de enfermagem sobre o...

\section{INTRODUÇÃO}

A atenção básica se caracteriza por um conjunto de ações, que visam à saúde do paciente de forma individual e coletiva com ações que privam em prover, prevenir e reabilitar o paciente de forma holística tem sua constituição uma estratégia na qual abrangem os princípios de universalidade, integralidade e equidade com atributos que tenha em vista a participação social integrada ${ }^{1}$. Os riscos de contaminação relacionado a profissionais de enfermagem são problema alarmante, são de fundamental importância as medidas preventivas em saúde e ações que repórter esses preceitos ${ }^{2}$.

As ações educativas em saúde entre a comunidade e Estratégia Saúde da Família de modalidade coletiva e individuais e caracterizadas como técnicas humanísticas por uma equipe multidisciplinar e interdisplinar com ênfase no profissional enfermeiro nas orientações e promoção da saúde ${ }^{1}$.

Os acidentes de trabalho (AT) envolvendo os profissionais de enfermagem vem tornando-se um dos grandes fatores para infecções devido as peculiaridades de atividades como manuseio de fluídos biológicos e exposições constantes a microrganismos, entretanto as infecções por usos inadequados de equipamentos de proteção individual (EPIs) constituem-se como grande problema de Saúde Pública ${ }^{3}$.

Entretanto de acordo com a literatura suscita reflexões sobre os riscos de infecções a acidentes de trabalho sendo que, a sobrecarga de trabalho predomina sendo um dos fatores determinantes nos acidentes ocasionados pelo uso inadequado de EPIs, estudos enfatiza a predominância em profissionais de nível médio em Enfermagem, pois, não se encontra uma formação que minimize esse índice de infecção a estimativa de infecção foi de $28,4 \%$.

Na Estratégia Saúde da Família, a educação em saúde de riscos de infecção pelo uso inadequado consiste em um dos principais elementos da promoção da saúde e, portanto, para que se consiga alcançar bons resultados, o processo educativo deve ser concentrado em ações e orientações que reportem a intenção em formar uma consciência saudável e não somente em trocas ou repasses de informações, que pouco contribui para o cuidado dessa população 5 .

Assim esse estudo tem como objetivo realizar uma reflexão sobre a formação do Enfermeiro no controle de infecção na estratégia na Estratégia Saúde da Família, no Município de Teresina, Estado do Piauí.

\section{METODOLOGIA}

Trata-se de um estudo descritivo e exploratório, de campo e de cunho qualitativo, primeiramente sobre o controle de infecção na Estrátegia Saúde da Família. Realizou-se um regaste histórico com referencial teorico sobre o controle de infecção na estratégia saúde família abordando os conceitos históricos e atuais da infecção na assistência nos serviços de saúde. Foi desenvolvida a discussão sobre a articulação desses conceitos na prática 
Cruz JN, Frota BC, Cruz FMN et al.

desenvolvida à abordagem a esses pacientes, tendo como foco nos estudante de enfermagem em uma instituição de caráter privado na área da saúde sendo que a pesquisa abora os conceitos nos quais a formação do estudante de enfermagem sendo relacionados às teorias de enfermagem no qual ver o paciente de forma holistica.

A análise crítica por afinidade de conteúdo das publicações se deu a partir da leitura e interpretação dos conhecimentos. O período de atualização bibliográfica ocorreu entre os meses de maio e agosto de 2015, com ênfase nas publicações dos últimos 8 anos, que apresentaram relevância com tema em questão referenciando os conceitos da enfermagem de forma geral.

\section{DESENVOLVIMENTO}

O papel da enfermagem no controle de infecção durante o século XIX, quando Florence Nightingale propôs a teoria do ambientalismo, sendo que a mesma condizem à importância do ambiente para a melhoria dos pacientes, Florence na guerra da criméia questionou sobre essa problemática do ambiente nas questões do bem estar e no prognóstico da doença, introduzindo posteriormente as técnicas de antissepsia, com a finalidade de diminuir os riscos de infecções, a profilaxia de infecção se desenvolve pelas as condutas de enfermagem nas atribuições do profissionais de evitar risco $\mathrm{IH}$, além de uma visão humanística profissionalizando a enfermagem ${ }^{6}$.
Formação do estudante de enfermagem sobre o...

Nessa contextualização histórica, enfatizase o quanto foi importante esse avanço, para a assistência ao paciente, com relação as teorias ambientalistas da teoria Florence onde o foco era o ambiente onde a mesma conceituava saúde como um processo reparador ${ }^{2}$. Pois, antes dela, os procedimentos realizados pelos profissionais eram todos feitos de forma empírica; ou seja, o conhecimento era adquirido no cotidiano, por meio das experiências, onde as técnicas não haviam embasamento científico; no período contemporâneo a saúde na atenção básica com ênfase na assistência de enfermagem segue de forma com medidas tecnológicas, na atualidade os processos tecnológicos computadorizados trazem melhorias na assistência ao paciente ${ }^{6}$.

Nessa vertente, as instituições de saúde concedem aos profissionais que prestam serviços nos órgãos de $\mathrm{CClH}$, desempenhar funções importantes para o conhecimento que adquiriram durante a graduação. Assim, os conhecimentos adquiridos durante a graduação exprimem as constantes importâncias para desempenhar um bom funcionamento na instituição de saúde, as técnicas de biosseguranças para as condutas nas instituições de saúde proporcionam as melhores condições para atuar o enfermeiro como medidas e as ações como técnicas corretas de uso de luvas, lavagem das mãos dentre outras abordam a importância e técnicas de controle de infecções ${ }^{7}$.

Com esses fatores, nota-se na literatura que a ocorrência de um processo infeccioso adquirido 
no ambiente dos serviços de saúde da atenção básica, na maioria das vezes, por fatores evitáveis. Como por exemplo: pela lavagem inadequada das mãos, manuseio de materiais e realização de técnicas desrespeitando os princípios de assepsia e falta de controle rigoroso no processamento dos materiais esterilizados, que vai desde a lavagem ao armazenamento e distribuição dos mesmos ${ }^{8}$.

A problemática da $\mathrm{IH}$, se caracteriza como um grande alarmante na saúde pública brasileira, que reportam a intenção de uma formação integral à sua prevenção. Sendo assim, envolve tanto as ações de enfermagem, como as condutas para minimizar os riscos de infecção. E nessa direção, essas ações se designam a observação entre pacientes hospitalizados ${ }^{9}$.

A formação de enfermeiros para atuarem controle se faz necessária para a melhoria de ações que regem o controle de infecção, os conhecimentos relevados com o resultado do presente estudo poderão servir para tomada de decisões para o profissional Enfermeiro nas intervenções de medidas educativas no controle de infeção na Estratégia Saúde da Família e possibilitar com o estudo a definição de medidas adotadas pelos Enfermeiros adquiridos com sua formação acadêmica ${ }^{8}$.

Entretanto faz a necessidade de aprimorar as medidas adotadas pelo os profissionais de Enfermagem no controle de infecções na Estratégia Saúde da Família, os mesmos estão voltados para utilização de técnica que possa melhorar esses fatores, por ações centradas em processos capazes de produzir de forma significativa melhorias nas áreas da saúde das famílias assistidas por esses enfermeiros, bem como aprofundar o conhecimento nesse âmbito ${ }^{10}$.

O estudo poderá contribuir no contexto de medidas profiláticas nos controles de infecção dos profissionais de saúde com ênfase no Enfermeiro que atua na Atenção Básica, possibilitando a construção de discussões e reflexões, além de novas produções teóricas que implicam na transformação de concepções e práticas gerando novos conhecimentos e saberes. Para a comunidade cientifica, o estudo torna-se extremamente importante e poderá ser utilizado como subsídio para embasar pesquisas futuras da mesma temática ${ }^{7}$.

Desta forma essa pesquisa busca elencar os padrões da formação de estudantes de enfermagem sobre sua trajetória acadêmica na enfermagem para o controle de infecção no ESF, sendo que é de crucial importância para o desenvolvimento. Os resultados deste estudo poderão servir como subsídios para a tomada de decisões pelo profissional docente da área da Saúde, fortalecendo o compromisso em aprimorar os conhecimentos sobre os estudantes de enfermagem sobre o controle de infecção e como lidar com essa problemática na atenção básica ${ }^{10}$.

A Estratégia Saúde da Família (ESF) surge como uma estratégia de viabilização dos princípios do Sistema Único de Saúde (SUS) de forma ágil e 
Cruz JN, Frota BC, Cruz FMN et al.

efetiva, na condição de que os profissionais envolvidos na construção e consolidação da Estratégia tenham habilidades para promover saúde, atuar na prevenção de agravos, diminuir os problemas sociais e de saúde da população atendida nos serviços de saúde ${ }^{8}$.

Entretanto faz a necessidade de aprimorar as medidas adotadas pelo os profissionais de Enfermagem no controle de infecções na Estratégia Saúde da Família, os mesmos estão voltados para utilização de técnica que possa melhorar esses fatores, por ações centradas em processos capazes de produzir de forma significativa melhorias nas áreas da saúde das famílias assistidas por esses enfermeiros, bem como aprofundar o conhecimento nesse âmbito 9

\section{CONSIDERAÇÕES FINAIS}

Os resultados fortalecem o compromisso de aprimorar o cuidado em saúde sobre o controle de infecção possibilitando que os enfermeiros que atuem em instituições de saúde reportem a intenção de adotar medidas que possam trazer melhorias para os pacientes vendo o mesmo como um todo resgatando nas teorias de enfermagem a importância, contribuindo no contexto da educação permanente dos enfermeiros que atuam na Saúde da Família, possibilitando a construção de uma pauta de discussões e reflexões, que implicam na transformação de concepções e práticas gerando novos conhecimentos e saberes.
Formação do estudante de enfermagem sobre o...

O estudo poderá contribuir no contexto da educação permanente na formação do estudante de enfermagem para lidar com o controle de infecção, possibilitando a construção de uma pauta de discussões e reflexões, além de novas produções teóricas que implicam na transformação de concepções e práticas gerando novos conhecimentos e saberes.

\section{REFERÊNCIAS}

1. Ministério da Saúde (BR). Sistema Nacional de Vigilância em Saúde: Relatório de Situação: Piauí. 5th ed. Brasília, 2011.Available drom: http://bvsms.saude.gov.br/bvs/publicacoes/siste ma_nacional_vigilancia_saude_pi_5ed.pdf

2. Haddad, VCNS; Franco, TC. A teoria ambientalista de florencenightingale no ensino da escola de enfermagem Anna Nery (1962 - 1968). Esc. Anna Nery [Impresso]. 2011[cited 2015 aug 10];15(4): 755-761. Available from: http: http://www.scielo.br/pdf/ean/v15n4/a14v15n4.pd $f$

3. Silva, CCS; Rodrigues, LMC; Silva, VKBA; Silva, ACO; Silva, VLA; Martins, MO. Percepção da enfermagem sobre condições de trabalho em unidades de saúde da família na Paraíba - Brasil. Revista Eletrônica de Enfermagem, [S.l.] 2013[cited 2014 Dec 16]; 15(1): 205-14. Available from:

http://revistas.ufg.br/index.php/fen/article/view $/ 15074$

4. Vieira, M; Padilha, MI; PINHEIRO, RIC. Análise dos acidentes com material biológico em trabalhadores da saúde. Rev. Latino-am. Enfermagem, 2011[cited 2014 Dec 16]; 8(19):1-14. Available from: http://www.scielo.br/pdf/rlae/v19n2/pt_15.pdf

5. Roecker S, Nunes EFPA, Marcon SS. Trabalho educativo do enfermeiro na Estratégia Saúde da Família: dificuldades e perspectivas de mudanças. Rev esc enferm USP [Internet]. 2012 June [cited 
indivíduo infectado. Rev Esc Enferm USP [Internet]. 2013[cited 2015 aug 10]; 47(4):860-8. Available from:

http: / / www.scielo.br/scielo.php?script=sci_arttext \&pid=S0080-62342012000300016

6. Vargas MA, Albuquerque GL Erdman ALR, Souza FR. Onde (e como) encontramos a qualidade no serviço de enfermagem hospitalar?. Rev. bras. enferm. [online]. 2007 60(3):339-343. Available from: http: / / www.scielo.br/scielo.php?pid=S003471672007000300018\&script=sci_arttext

7. Viera, M; Padilha, M; PINHEIRO, RC. Análise dos acidentes com material biológico em trabalhadores da saúde. Rev. Latino-am. Enfermagem Enfermagem. [online]. 2011[cited 2014 Dec 16]; 8(19):1-14 Available from: www.eerp.usp.br/rlae

8. Fracolli LA; Zoboli ELP; Granja GF; Ermel RC. Conceito e prática da integralidade na Atenção Básica: a percepção das enfermeiras. Esc Enferm Usp. [online]. 5 (45): 1135-1141.

9. CostaFM, Martins AMEBLM, Santos Neto, PE, Veloso DNP, Magalhães VS, Ferreira RC. Is

\section{Colaborações}

Nascimento JC, Frota BC, Almeida CAPL contribuíram na percepção e planejamento do trabalho e na análise e interpretação dos resultados obtidos. Calland FN, Cruz FMN e Lima CHR, contribuíram na elaboração e organização das ideias para formação do trabalho e das revisões sucessivas até a aprovação final. vaccination against hepatitis $B$ a reality among Primary Health Care workers? Rev Latino-Am. Enfermagem. [Internet]. 2013[cited 2014 Dec 16] Feb; 21(1):316-324.

10. Formação do Enfermeiro na prevenção da hepatite $\mathrm{B}$ : análise de similitude e nuvens de palavras. Coêlho LS, Carvalho LRB, Sousa BSA, Cruz JN, Almeida CAPL, Lino MM. Rev Pre Infec e Saúde [Internet]. 2015 [cited 2015 aug 10]; (1):31-8. Available from: http://www.ojs.ufpi.br/index.php/nupcis/article/ view/3624 